\title{
Detection of Body Movement for Comatose Patient Using IoT
}

\author{
Nisha. $Y^{\mathrm{a}, 1}$ and K.Valarmathi. ${ }^{\mathrm{b}}$ \\ a PG Scholar, Dept of CSE, Panimalar Engineering College, Chennai, India \\ ${ }^{\mathrm{b}}$ Professor, Dept of CSE, Panimalar Engineering College, Chennai, India
}

\begin{abstract}
Coma is the state of anesthetized or unconscious state where the patient doesn't respond to the actions or any activities. Since the patients are in unconscious state the patients at tender or the doctors don't have time to observe the patient activities continuously. In order to overcome this problem an IoT based system is designed to monitor the body movements and the actions of the coma patient using various sensors. The equipment has been planned that utilizes Arduino controller board. In addition with the Arduino board various sensors have been associated through the controller board interface. The sensors values will be transferred to the cloud server. The movement of eye ball retina and motion of the body detected by the wearable motion sensors state are monitored. By using the sensors it is helpful for the doctor and the specialist about the well-being condition of the patients and continuously monitoring their physical condition. The challenging task is focused on the limitation of the existing techniques are discussed.
\end{abstract}

Keywords.IOT, Arduino controller, sensor, cloud server, sensors.

\section{Introduction}

Coma is a state of oblivion in which a human being cannot be awaken; fail to take action on average to agonizing stimuli, light, or sound; lacks abnormal wake sleep cycle, and does not begin voluntary actions. Coma might occur for several reasons, such as intoxication, a disease or infection that affects the central nervous system (CNS), a serious injury, and hypoxia, or oxygen deficiency. Doctors will monitor the activity of the patient continuously in order to find the improvement in patient health. Also they will monitor the coma person's body movements and position, response to painful health status or stimuli, and pupil size. Doctors will monitor and observe the nasal breathing pattern that helps in diagnosing the cause and reason of the coma.

\footnotetext{
${ }^{1}$ Y.Nisha, PG Scholar, Dept of CSE, Panimalar Engineering College, Chennai, India,

E-mail: nishayuva4@gmail.com
} 
In order to determine the coma affected person's stage of consciousness. Doctors will regularly monitor the signs of arousal, such as voice, vocal noises, eyes pupil motion or movement. And also will test and test the reflexive eye actions. All these tests may help in finding out the cause of the coma and the spot or the region of brain failure or damage. The system has been designed such that the patient under the treatment is completely under surveillance so that all the information of the patient is directly stored in the server. For monitoring the patient physically it is usually happening in hospitals where two or three hospital staff needed to monitor the patient $24 * 7$ for surveillance if there is movement or not, but this is not well-organized method to get maximum efficiency. So this project helps to monitor the patient physically for every moment. The system is developed based on motion Flex sensor, Eyeball pupil sensor, MEMS or accelerometer body sensor, Heartbeat rate sensor, Temperature sensor and the Pressure sensor which is used to develop and design a system that will monitors the body movement of the person at coma stage and alerts automatically if any abnormal activity occurs by sending a message to the respective person using IOT.

\section{Related works}

K.Vishwanatha [1] has proposed a model that is reliable, precise and smart with robust algorithm that helps in monitoring the motion detection of the patient. This system uses algorithm called as background subtraction. A portable system that consist of a test kit which associates in detection of eye blink for comatose patient was developed by [2]V.Subharamya This system provides the physical health monitoring phase. ShwetaS.kshirsagar[3]enabled a model that provides the interaction sensors and the cloud server. It provides an communication module to the patient who is in coma and the relative of the patient about the health status of the patient. A model has been developed in order to detect the changes of the patient who is in the comatose state.Yuvaraju.M and priyanka.R [4] has suggested the model based on the flex sensors. A study has conducted in the year of 2013 by GuhaBalakrishnan[5].this system extracts the heart beat rate and analyze the rate by Newtonian method. Also this captures the data clinically and sends the data to the cloud system. A study conducted in the year of 2020 based on the development of Multi-parameter system for detecting patient health monitoring system by Athira.A et al [6]. Zakir Husain et al [7] has designed a system that helps in continuous monitoring of the health status of the comatose patient based on the internet of things .the abnormal movement captured in acutely ill patient with the major brain injuries. Muhammad Naufal Bin Mansur et al [8] has proposed a system using K-NN and LDA algorithm for expression analysis under different lighting for coma patients. Naveen Kansal, Hardeep Singh Dhillon[9]developed a system that provides the interface between the sensor hub and the cloud server all the data of the patient are controlled and preserved for the emergency situations.V. Ferrari[10]enabled a system that helps in acting as a tool for monitoring the coma patient viral parameters such as heart rate, pulse rate and all the day will be stored in the cloud server. If the system reaches the threshold value then the system will alert through the message or email [11-18]. 


\section{Methodology}

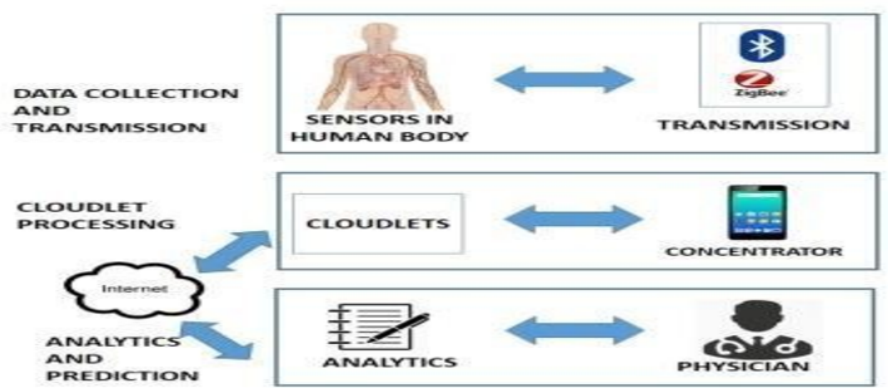

Figure 1. Proposed System Framework

Figure 1.represents the overall proposed system framework. The health monitoring system proposed consists of several and variety of sensors that are alienated into two categories. The first category is mainly used in order to monitor the vital signs of the coma patient and second component is mainly used in order to detect the changes physically that occurs in the coma patient. In this system, temperature, heart rate, movement of the patient, blood pressure are the major vital information that has been monitored, preserved and recorded in order to be aware of status of a health of coma patient. The FLEX sensor and PIR sensor are mainly used for in order to detect the changes bodily like movement of the body that occurs in a coma patient body. All these signals helps in providing the information that are recorded and monitored continuously in order to understand the functioning of the body. ECG Sensor is mainly used to monitor the of heart beat. If the signals are sensed which are outside the threshold value or the normal values ranges naturally that implies the need for emergency care or possible evacuation to provide a higher level of treatment during which the system alerts the care taker and also the doctor.

\section{Data flowdiagram}

\subsection{Level 0}

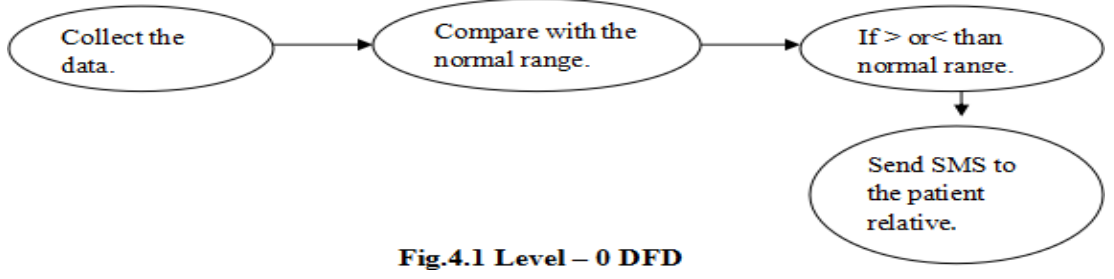

Figure 2. Level - 0 DFD

The figure 2 shows the DFD diagram for level 0.This DFD level 0 diagrams represents the complete process of the system. This diagram represents collection of data from the 
sensor to the IOT board these data are processed in the microcontroller and then if any abnormal range occurs then it will immediately send the message to the respective patient relative.

\subsection{Level 1}

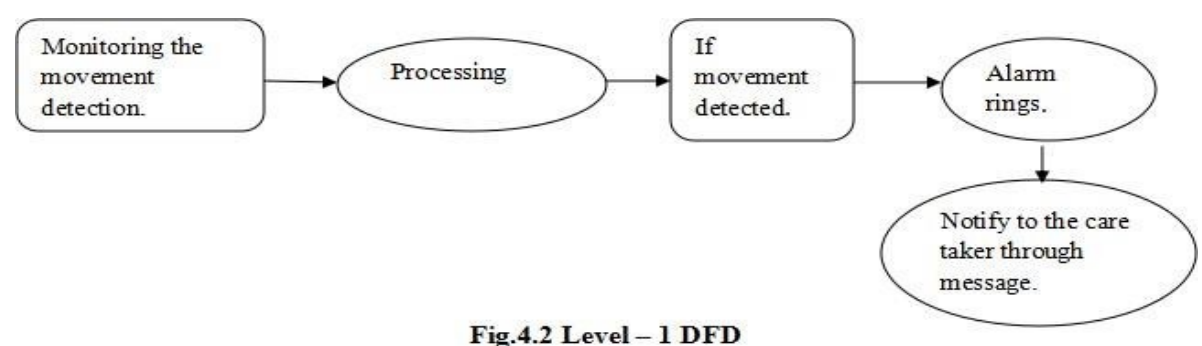

Figure 3. Level - 1 DFD

The figure 3 represents the Level - 1 DFD which extends the process of movement detection. The sensor data is given as an input to the system where is it processed and then monitors the changes in the movement of the body. If any changes occur in the body then the data is updated in the database and also the alarm is received to the care taker.

\section{Conclusion and futurework}

The endeavor of our planned system is to build easily easy to get to design that the patient's information is stored and easily monitored by the doctors and staff. This planned model helps in several ways that can be easily retrieve the data of the patient that has been stored in the server. So this system provides the maximum efficiency and high throughput. All the critical information of the patient is conveyed quickly to the doctor is achieved. The designed model leads to the better and effective health care service to comatose and the collected data is networked worldwide with the help of internet and communication which provide a quickresponse.

\section{References}

[1] K. Vishwanatha ,Murigendrayya M. Hiremath, Advances Motion Detection Algorithm for patient monitoring using cell phone with video display, international journal of electronics signals and systems volume 2 , issue 3.

[2] V.SubhaRamya,AmeerVilangali, portable test kit for coma patient with eye blink alert system, International Journal of Advanced Research in Electrical, Electronics and Instrumentation engineering.vol.7, issue4.

[3] ShwetaS.Kshirsagar,P.A.Ghone, Movement detection using image processing, International Journal of Science and Research (IJSR) Volume 4 Issue 2, February2015.

[4] Yuvaraju.M and priyanka.R, Flex sensor based gesture control wheelchair for stroke and SCI patients, International Journal Of Engineering Sciences \& Research Technology,May2017. 
[5] GuhaBalakrishnan,FredoDurand,JohnGuttag .Detecting pulse from head motions in video. IEEE xplore vol23,issue3.

[6] Athira.A, Devika.T.D, Varsha.K.R, SreeSanjanaaBose.S .Design and Development of IOT Based Multi Parameter Patient Monitoring System. International Conference on Advanced Computing \& Communication Systems,2020.

[7] ZakirHussain, Ravindiran, Health Monitoring System for Comatose Patients: A Survey, International JournalofAdvanceResearch,IdeasandInnovationsinTechnology, Volume 3, Issue6,2017.

[8] Muhammad Naufal Bin Mansur, Smart Health Monitoring Systems: An Overview of DesignandModeling, Journal of Medical System, Vol.37,2013.

[9] Naveen Kansal, Hardeep Singh Dhillon, Advanced Coma Patient Monitoring System, International Journal of Scientific \& Engineering Research Volume 2, Issue6.

[10] V. Ferrari, T. Tuytelaars, and L. V. Gool, Real-time affine region tracking and coplanar grouping, in Proc. IEEE Conf. on Computer Vision and Pattern Recognition, Hawaii, vol. II,2001.

[11] V.D.AmbethKumar ,S.Ramya , H.Divakar, G.KumuthaRajeswari .A Survey on Face Recognition in Video Surveillance. Lecturer Notes on Computational and Mechanism, Vol. 30, pp: 699-708, 2019

[12] Ambeth Kumar.V.D .Precautionary measures for accidents due to mobile phone using IOT. Clinical eHealth, Volume 1, Issue 1, March 2018, Pages 30-35.

[13] Nanagasabapathy.K ,G. Harish; O. I. Allen Sebastian; N. Sowrabh Chandra; V. D. Ambeth Kumar. Validation system using smartphone luminescence.IEEE International Conference on Intelligent Computing, Instrumentation and Control Technologies (ICICICT), Pages: 235 - 239, 6-7 July 2017 , Kannur, India

[14] Ambeth Kumar.V.D, V. D. Ashok Kumar; H. Divakar; R. Gokul. Cloud enabled media streaming using Amazon Web Services. IEEE International Conference on Smart Technologies and Management for Computing, Communication, Controls, Energy and Materials (ICSTM), Pages: $195-198$, 2-4 Aug. 2017, Vel Tech University, Chennai, India (DOI: 10.1109/icstm.2017.8089150)

[15] Aravindh.B, V.D.Ambeth Kumar; G. Harish; V. Siddartth. A novel graphical authentication system for secure banking systems .IEEE International Conference on Smart Technologies and Management for Computing, Communication, Controls, Energy and Materials (ICSTM), Pages: 177 - 183, 2-4 Aug. 2017, Vel Tech University, Chennai, India

[16] Ambeth Kumar.V.D et.al, (2016).An Efficient Security System for Data base Management from Illegal Access. IEEE International Conference on Wireless Communications, Signal Processing and Networking (WiSPNET), SSN Engineering College, Chennai, India, 23-25 March, 2016

[17] Ambeth Kumar.V.D and Dr.M.Ramakrishan (2011).Web Cam Motion Detection Surveillance System Using Temporal Difference and Optical Flow Detection with Multi Alerts. (CCIS) Journal (Springer) Volume 147, Part 3,pp: 214-221, 2011, April 2011 and the paper was published in the International Journal. [Impact Factor: 0.247].[DOI: 10.1007/978-3-642-20573-6_34]

[18] Ambeth Kumar.V.D and Dr.M.Ramakrishan (2012). Enhancement in Footprint Image using Diverse Filtering Technique. in the month of March for the Procedia Engineering journal (Elsevier) Journal Volume 8, No.12, 1072-1080, March 2012 and the paper was published. [doi:10.1016/j.proeng.2012.01.965]

[19] R. Subha Shini et.al., " Recurrent Neural Network based Text Summarization Techniques by Word Sequence Generation",IEEE International Conference on Inventive Computation Technologies (ICICT), 2021, DOI: 10.1109/ICICT50816.2021.9358764

[20] S.V. Ruphitha et.al, “ Management of Major Postpartum Haemorrhage by using Zigbee protocol - A Review “, 2021 6th International Conference on Inventive Computation Technologies (ICICT) (DOI: 10.1109/ICICT50816.2021.9358757)

[21] M. Indhumathi et.al , "Healthcare Management of Major Cardiovascular Disease-A review", 2021 6th International Conference on Inventive Computation Technologies (ICICT), (DOI: 10.1109/ICICT50816.2021.9358519)

[22] .K. Sabarinathan et.al ., " Machine Maintenance Using Augmented Reality”, 3rd International Conference on Communication and Electronics Systems (ICCES), 2018. (DOI: 10.1109/CESYS.2018.8723900) 